\title{
Performance Comparison of Routing Protocols in Opportunistic Networks
}

\author{
Chander Prabha ${ }^{1}$, Rakesh Kumar ${ }^{2}$ \\ ${ }^{1}$ Associate Professor, Department of Computer Science and Engineering, \\ ${ }^{2}$ Professor, Department of Computer Science and Engineering, \\ Chandigarh University Gharuan Mohali Punjab, India \\ 'prabhanice@gmail.com, 2.Rakesh77kumar@yahoo.com
}

\begin{abstract}
In today's world doing data transfer in delay tolerant networks (DTN) environment is a challenging task. In DTN nodes are characterized to meet opportunistically to do routing and data transfer. In opportunistic environment no end-to-end path exists between destination and source. The contacts are made opportunistic while coming in contact for a short span of time. All communication is within this span only. Due to this feature the DTN's are sometimes recognized as Opportunistic Networks (ON's). The rules are not predefined here for choosing the next node as applicable in conventional schemes of routing. In this paper the performance of opportunistic routing protocols have been investigated namely PRoPHET, Spray and Wait, SimBet, Bubble Rap in terms of robustness and scalability. The concept of Ant Colony Optimization is used to find optimal routes while doing routing decision. The performance of SimBet and Bubble Rap is better with respect to throughput as they belong to social context aware category of protocols. Performance is evaluated in terms of packet dropped and overhead ratio also. The overhead ratio is better in SimBet and Bubble Rap as compared to Spray and Wait and PRoPHET. Depending on buffer size, speed, contact times these routing strategies shows variable performance. The result indicates that the social aware algorithms have the ability and capacity to exchange/carry information faster and improve the connectivity in $O N$ 's.
\end{abstract}

Index Terms-DTN's, ONE Simulator, ON's, ACO, Centrality.

\section{INTRODUCTION}

$\mathrm{I}$ $\mathrm{N}$ MULTI-HOP Ad-hoc networks the communication is possible even if a direct route doesn't exist between source and destination. The communication is provided through opportunistic links. These networks are prone to long delays and therefore a part of delay tolerant networks (DTN's). In opportunistic environment strong connectivity is not a requirement. These networks uses store and carry forward concept. ON's are characterized as topology-less, no direct path, long disruptions and partitioning lasting for long periods. The idea of opportunistic networks was proposed by Kevin Fall [1] in 2007 as an extension of DTNs with an assumption of more unpredictable mobility [2]. The nodes came in contact opportunistically without any previous information. The features of ON's result in longer delays than conventional networks employed by MANETs. The existence of ON is shown in Fig. 1.

The characteristic of opportunistic network are:

- Power constrained devices,

- Intermittent connectivity,

- Occasional contacts,

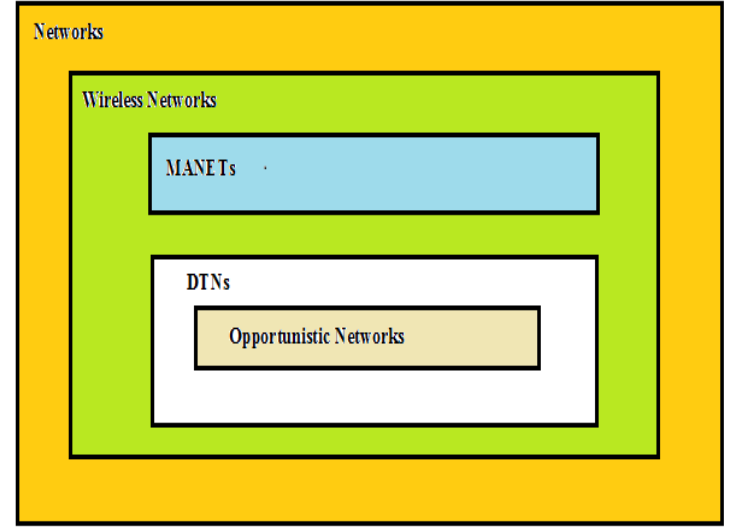

Fig. 1. Opportunistic Networks

- Highly mobile nodes,

- Non-existence of end-to-end paths.

The communication range of devices carried by humans, vehicles etc. are very short in opportunistic. Due to powerconstrained and limited communication range of devices, it is sometimes tough to establish and preserve the connection and communication from source to sink. ON's are the one of the most interested evolution of MANET. The essential requirement of MANET is that the both source and sink nodes must be within the range at the same time and should be connected to the network, when sender wants to send the message and if the sink is not connected to the network messages get dropped at some point of network. ON's aim is to allow user enable to pass and receive data/information even in such a disconnected scenario. In ON's, nodes are able to do communication even if there is no route exists that connects. Routes are dynamically built, while messages are on their way to route between the source and the $\operatorname{sink}(\mathrm{s})$. Any encountered possible node can opportunistically be used as next hop, provided it is likely to bring the message nearer to the final sink. ON a promising and challenging research field due to these requirements. The design of efficient routing strategies for ON's is generally a complicated task due to the absence of knowledge about the topological evolution of the network. Fig.2. shows the hierarchy of communication networks.

\section{A. Challenges and issues in ON's}

Following are the challenges in opportunistic networks. 
- Forwarding and routing of data

- Buffering

- Link Discovery

- Contact opportunity

- $\quad$ Security and privacy

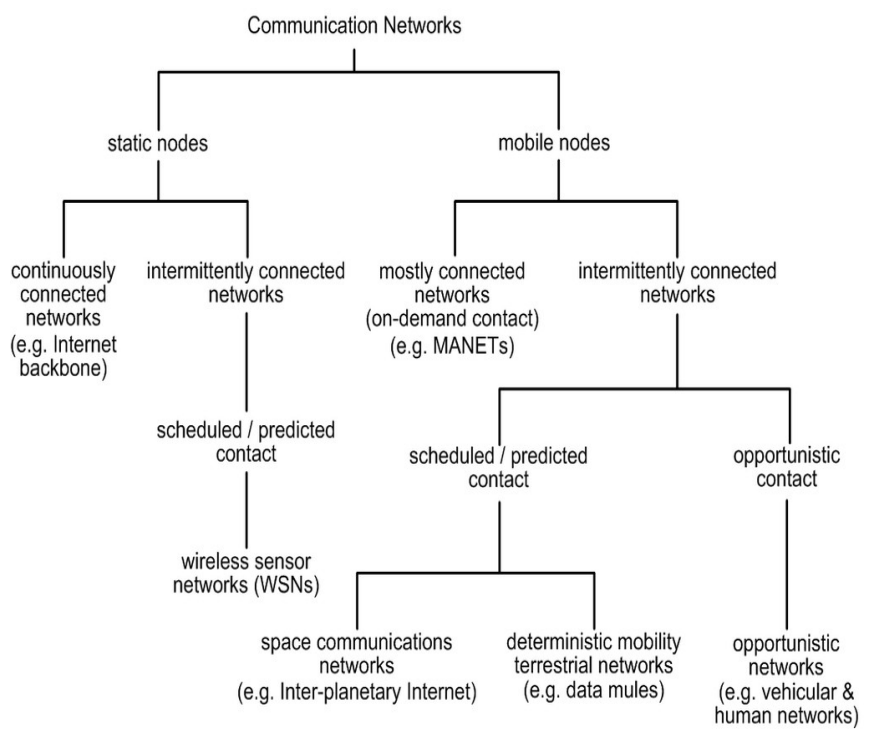

Fig .2. Hierarchy of Networks

The environment of opportunistic network is disconnected. The connectivity of nodes is sporadic. The sporadic connectivity of nodes limits the node contribution in forwarding the data and consequently affecting the performance of routing protocol. No information about the topology of network is available. All these challenges play a vital role and must be taken care of while designing and efficient routing protocol in opportunistic networks [3]. Section 2 describes routing protocols for performance comparison purpose in opportunistic networks.

The objectives of the results presented in this paper is to show that social context aware routing protocols have better performance than mobility based category of routing protocols in opportunistic networks. The performance of four protocols have been investigated and discussed in terms of metrics viz. throughput, packet dropped and overhead.

\section{RELATED WORK}

This section presents review on common routing protocols in ON's under consideration in this paper. Most common approach of routing is presented by Vahdat and Becker [4] called Epidemic. The nature of this protocol is floodingbased and is under context-oblivious category of routing protocol in ON's. Here nodes replicate continuously and messages are transmitted to newly revealed nodes with a condition that they do not already retain a message copy. The messages are delivered ultimately to destination based on pair-wise information maintained by nodes on encountering each other. However if path to sink is not available then node will buffer/hold the message in summary vector index. Every message has a unique ID to avoid duplicity. To manage node resources utilization to be low, a hop counter is associated with each message that it can travel to.

Probabilistic routing algorithm called PRoPHET is presented by [5] Anders Lindgren and et al. PRoPHET stands for Probabilistic Routing Protocol using History of Encounters and Transitivity. It falls under mobility based category of routing protocols in ON's. The method adopted by PRoPHET is similar to Epidemic, here also nodes want to communicate exchange summary vectors (SV) when they meet. Additionally summary vectors of PRoPHET contains the delivery predictability information (DPI). This information is stored in the nodes. The internal delivery predictability vector is updated using DPI and then the information contained in the SV serve the purpose of deciding which messages to request from the other node. Here the node forwarding lean on the DPI of the nodes come across. This predictability of reliable node is an important parameter in PRoPHET. It is used to decide which node is more positive and trusty than the other for doing forwarding of messages to the destination node.

Thrasyvoulos Spyropoulos and et.al. proposed Spray and Wait protocol (SW) [6]. It consist of two phases: 1) spray phase and 2) wait phase. The SW protocol are part of mobility based category of routing approaches in ON's. In this routing approach on generation of any new message in the network, a number $\mathrm{A}$ is appended to the message, where $\mathrm{A}$ indicates maximum admissible replica of message in the network. In the first phase, spraying of message is done by originate node, after receiving the message by intermediate node, the second phase, wait activates where the intermediate node stores that particular message until the sink encountered directly.

The next two routing protocols (SimBet and Bubble Rap) under consideration are under Social context aware protocols in opportunistic networks. SimBet [7] uses the concept of Between-ness centrality that tells how much a node is interconnected. This algorithm is based on forwarding messages to nodes with having larger value of SimBet relevance. For reducing overheads it uses local centrality metric and between-ness metric. In order to make the forwarding decision SimBet exchanges the pre-estimated centrality and locally determined similarity of each node.

Bubble Rap [8] make use of two supposition a) every node must pertain to at least one social community. The popularity of that node is described by its centrality within its community (i.e. local centrality etc). b) There is a local ranking (local centrality) and global ranking of every node (i.e., global centrality) within its community and in the whole system respectively. The node may have several local centralities if node belongs to several communities.

Now the work has been extended for saving the energy of nodes. EEOR-FL [13] routing focuses on to balance energy consumption while maintaining lifetime of network. It uses a method to calculate the candidate list. 


\section{Simulation Strategy}

In the present paper, simulations are done to analyze the performance of mobility based (PRoPHET and Spray and Wait) and social context aware (SimBet and Bubble Rap) based routing protocols using opportunistic network environment simulator (ONE version 1.4.1) [9]. This tool is used in ON research. It is based on JAVA environment that supports variety of mobility models. The ON are modeled as dynamic group of nodes (mobile) which joins or leave the group at any time. Five groups of moving nodes are taken viz. light weight vehicles, pedestrians, trams, bicycle and office workers. These groups follows Map based movement model to reach from one place to another with different speeds. Metrics for evaluating the performance of routing protocols are throughput, packet dropped and overhead ratio. Points followed while evaluating and getting the metrics results are:

i) ACO is used for doing routing decision. ACO is a metaheuristic technique [10]. It finds solutions to combinatorial optimization problems and is an iterative distributed algorithm.

ii) Social information of the node is gathered by use of $\mathrm{Be}$ tween-ness centrality which shows how much packet delivers and drops in a network from source to destination [12].

iii) The shortest path is finding out by using Dijkstra algorithm [11].

This paper uses ACO [14] [15] to optimize the multiple routes while searching the path. It depends upon behavior of real ants and having reinforce abilities viz. quickly recognizing the followed routes and knowledge about the distance to other routes. The parameter of concern of routing for optimization here is the between-ness centrality that describe the most important node in the network. The reason for choosing ACO algorithm [16] is specially it helps in propagation process at a faster rate.

\section{RESULTS}

Throughput: It is the defined as rate of successful message received/delivered. Fig. 3 shows throughput versus number of nodes of the four routing protocols. The throughput of social based routing algorithms are higher than the mobility based.

Dropped Packets: The reason for occurrence is network congestion. Mainly loss of packets occurs when more than one packet get into the network over a medium and fails to reach their corresponding destination. Fig. 4 shows no of packet dropped w.r.t four protocols. In terms of increase in nodes the packet drop decreases in SimBet and Bubble Rap, whereas it increases in case of PRoPHET and Spray and Wait. The reason for decrease in packet drop is due to increase in social interaction and optimization of ACO [16] during propagation process in case of social context aware routing protocols, whereas it is not the case with other two.

Overhead Ratio: Shown in Fig. 5. Calculation is done by using number of packet delivered, received at a node and number of packet dropped. It is less in SimBet and Bubble

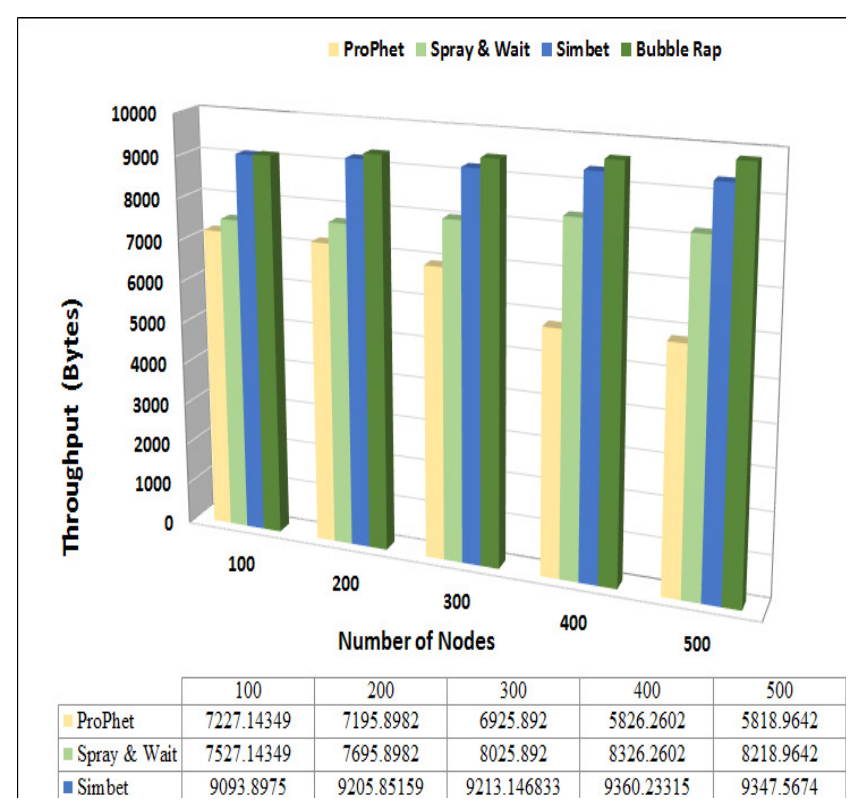

Fig. 3. Throughput versus Number of Nodes

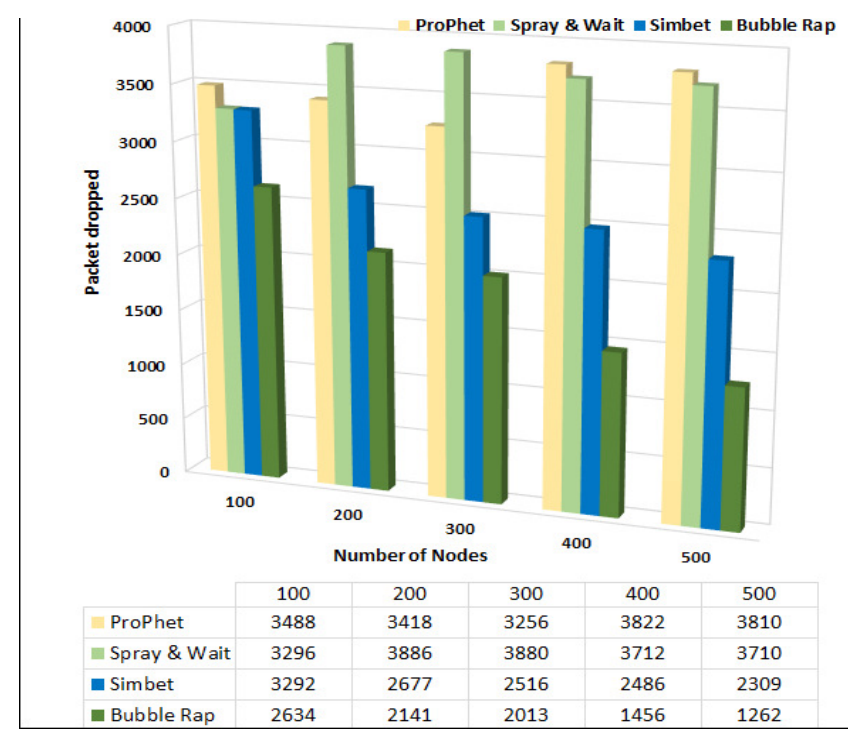

Fig. 4. Packet dropped versus Number of Nodes

Rap as compared to PRoPHET and Spray and Wait. The ratio of overheads also increases as nodes increases in the network.

\section{CONClusion AND Future Scope}

In ON's connectivity in intermittent due to which spontaneous data transmission is required as and when connectivity become available. In this paper with the use of ACO optimization an attempt is made to improve the throughput of a network. By using between-ness centrality the packet drop rate and overhead ratio is reduced. In routing where nodes are themselves carriers then nodes in the network are also data collectors. Nodes are following either arbitrary or predetermined routes around in the network area to move. 


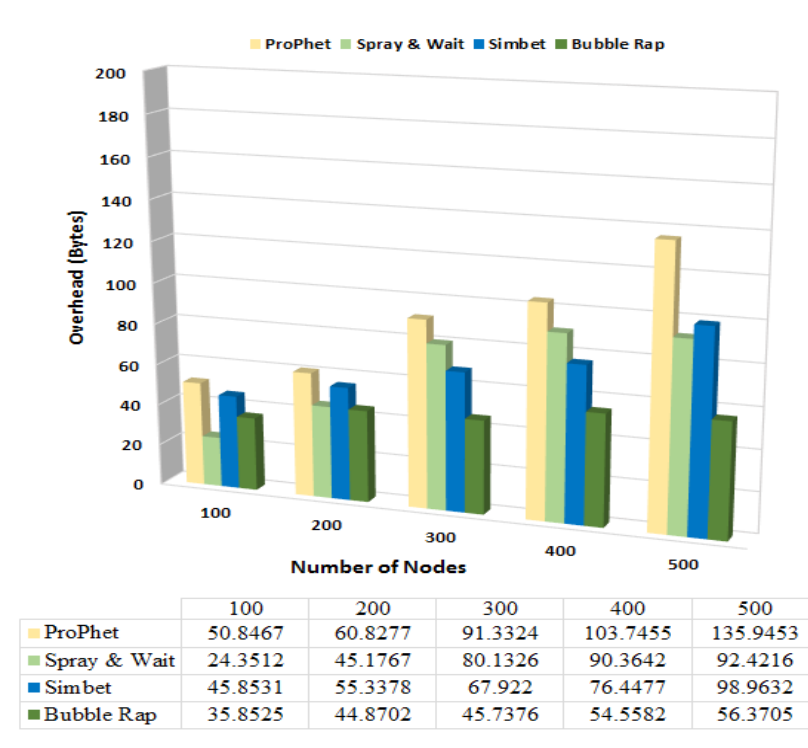

Fig. 5. Overhead Ratio versus Number of Nodes

Moving of nodes required to gather messages from the encountered nodes. These can be the only entities culpable for delivery of messages. This paper concludes that routing done through mobility based approach is not delivering better throughput as compared to social based. The packed dropped rate is also higher and it tends to increase wr.t scalability in mobility based (Spray and Wait and PRoPHET). The ration of overhead is also better in social based (SimBet and Bubble Rap). The work can also be enhanced by evaluating these protocols with respect to other parameters like buffer constraints, delay, and energy saving techniques.

\section{REFERENCES:}

[1] Chung-Ming Huang, Kun-chan Lan, "A Survey of Opportunistic Networks", 22nd International Conference on Advanced Information Networking and Applications, March 2008.
[2] Kevin Fall, "A Delay-Tolerant Network Architecture for Challenged Internets" SIGCOMM'03, August 25-29, 2003, Karlsruhe, Germany.

[3] Opportunistic Networks: Present Scenario-A Mirror Review, Article (PDF Available) in International Journal of Communication Networks and Information Security 10 (1) April 2018.

[4] Vahdat, A., \& Becker, D. (2000), Epidemic routing for partially connected ad hoc networks.

[5] A. Lindgren, A. Doria, and O. Schelen, "Probabilistic routing in intermittently connected networks. SIGMOBILE Mob," Comput. Commun. Rev. vol. 7, no. 3, 2003.

[6] T. Spyropoulos, K. Psounis and C. S. Raghavendra, "Spray and wait: an efficient routing scheme for intermittently connected mobile networks", In WDTN'05: Proceedings of the 2005 ACM SIGCOMM workshop on Delay-tolerant networking, 2005.

[7] P. Hui, J. Crowcroft and E. Yoneki, "Bubble Rap: Social based Forwarding in Delay Tolerant Networks," Proceedings of the ACM International Symposium on Mobile Ad hoc Networking and Computing, Hong Kong, China, May 2008.

[8] M Shah et.al., "An Improved SimBet Routing Algorithm for Human Mobility based DTN", Kalpa Publications in Computing Volume 2, 2017, Pages 166-176.

[9] A. Ker Anen, "Opportunistic network environment simulator. Special assignment report, Helsinki university of technology," Department of Communications and Networking, May 2008.

[10] Marco Dorigo, Luca Maria Gambardella, "Ant colony system: A cooperative learning approach to the traveling salesman problem" IEEE transaction on evolutionary computation, vol. 1, No. 1, April 1997.

[11] DongKai Fan, Ping Shi, "Improvement of Dijkstra's Algorithm and Its Application in Route Planning", Seventh International Conference on Fuzzy Systems and Knowledge Discovery, 2010.

[12] Payal Jhadhav, Prof. Rachna Sarao, "A Survey on Opportunistic Routing Protocols for Wireless Sensor Networks", Procedia Computer Science 79(2016), 603-609.

[13] Hajer Ben Gradz et.al, "Opportunistic Routing Protocols in Wireless Sensor Networks", Wireless Personal Communications, 104, (921933), 2019.

[14] Taku Yamazaki et.al, "Ant-inspired Backoff-based Opportunistic Routing for Ad Hoc Networks", 2019 20th Asia-Pacific Network Operations and Management Symposium (APNOMS), Sep 2019.

[15] S.Harikhore et.al.' "Ant colony optimization based energy efficiency for improving opportunistic routing in multimedia wireless mesh network", December 2019, Indonesian Journal of Electrical Engineering and Computer Science 16(3):1371

[16] Raghu Ramamoorthy, Menakadevi Thangavelu, "An improved distance-based ant colony optimization routing for vehicular ad hoc networks", International Journal of Communication Systems, June 2020. 